Maximilian Hoor, Eva Fraedrich, Charlotte Räuchle, Robert Kitzmann

\title{
Diskurse, Räume, (Online-)Medien. Eine Methodendiskussion anhand empirischer Beispiele
}

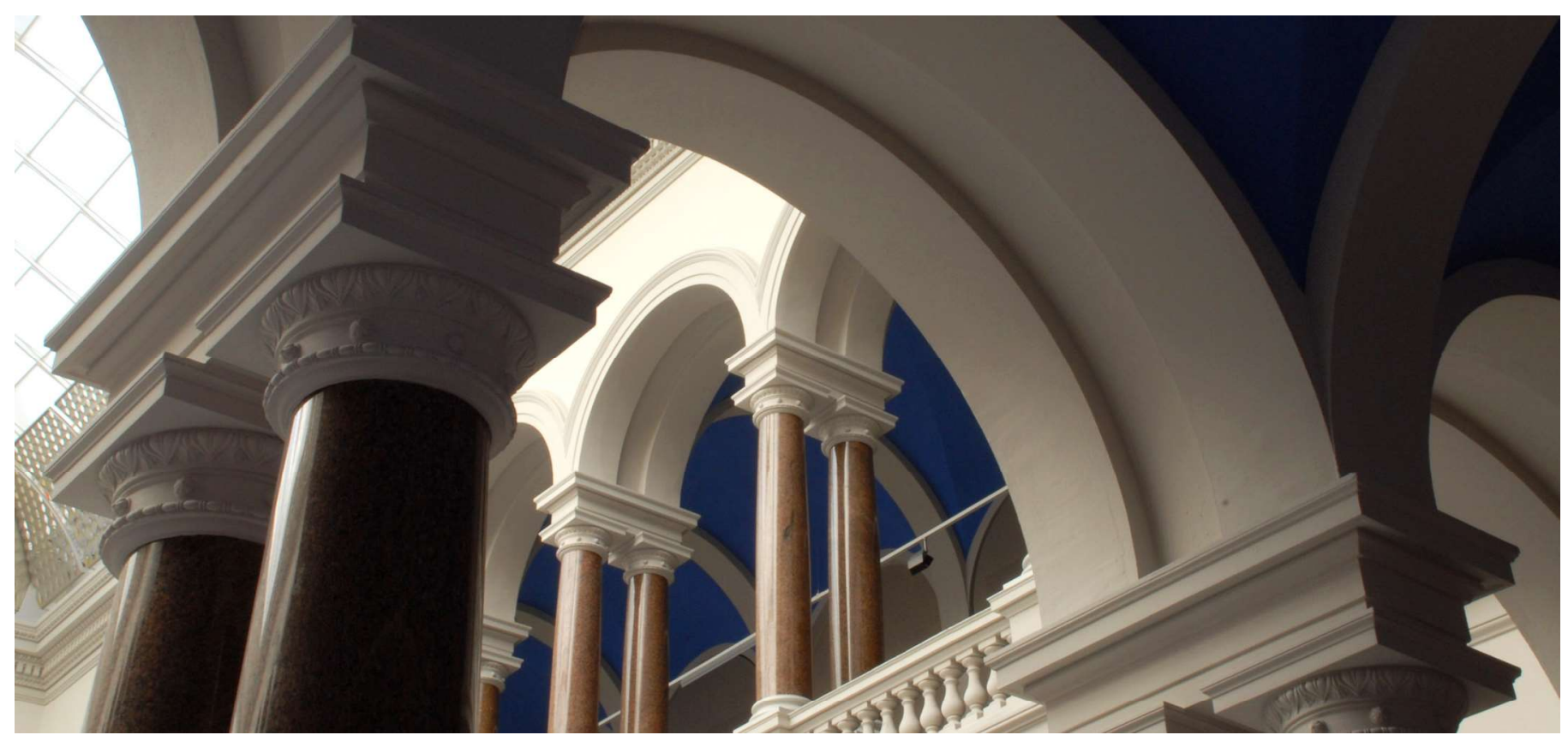

Hoor, M., Fraedrich, E., Räuchle, C., \& Kitzmann, R. (2018). Diskurse, Räume, (Online-)Medien. Eine Methodendiskussion anhand empirischer Beispiele. In Sozialraum erforschen: Qualitative Methoden in der Geographie (pp. 229-244). Springer Berlin Heidelberg. https://doi.org/10.1007/978-3-662-56277-2_15 


\section{Diskurse, Räume, (Online-)Medien. Eine Methodendiskussion anhand empirischer Beispiele}

Maximilian Hoor, Eva Fraedrich, Charlotte Räuchle, Robert Kitzmann

\section{Zusammenfassung}

Der Beitrag diskutiert, wie textbasierte Diskurse aus einer humangeographischen Perspektive analysiert werden können. Dabei wird auf der Grundlage aktueller Debatten zur Besonderheit internetbasierter Daten und Kommunikation deren Einfluss auf die Entwicklung der raumbezogenen Diskursanalyse erläutert. Beispielhaft werden diese Aspekte anhand des Online-Mediendiskurses zum jährlich stattfindenden Kunstfestival 48 Stunden Neukölln diskutiert. Das methodische Vorgehen wird detailliert beschrieben und schrittweise vorgeführt, wie eine Kritische Diskursanalyse zur journalistischen Berichterstattung und ihrer Online-Rezeption anhand einer aktuellen sozialräumlichen Fragestellung praktisch umgesetzt werden kann.

\subsection{Einleitung}

Diskursanalytische Verfahren, die sich an die (de-)konstruktivistische Diskurstheorie Foucaults (1981) anschließen, haben innerhalb der sozialwissenschaftlichen Forschung in der Vergangenheit viel Aufmerksamkeit erfahren und sind mittlerweile ein fest etablierter Bestandteil des Methodenkanons (vgl. u. a. Dreesen et al. 2012; Jäger 2009; Jäger und Jäger 2007; Fairclough 1995). Im Zuge des spatial turn werden diskursanalytische Verfahren auch in der raumbezogenen Forschung genutzt, um Räume und Orte in Bezug auf ihre (macht-)politische Bedeutung zu hinterfragen. Ziel dieser Arbeiten ist, Bedeutungsstrukturen und Zusammenhänge von Diskurselementen aufzuspüren und nach deren Rolle für die Produktion einer Wirklichkeit zu fragen (vgl. Glasze und Mattissek 2009). Eine besondere Relevanz hat dabei die Untersuchung textbasierter, speziell journalistischer Diskurse: So wirken etwa Interdiskurse in massenmedialen Printmedien auf die Konstituierung des Alltagswissens einer breiten Bevölkerung ein und können zumindest als ein Faktor gesellschaftlicher Meinungsbildung identifiziert werden (vgl. Fairclough 2000). Nicht alle-wissenschaftlichen Diskurse sind Interdiskurse - diese grenzen sich z. B. von sogenannten Spezialdiskursen (etwa Wissenschaftsdiskurse) ab, indem sie als eine Art Bindeglied derselben fungieren, mit deren Hilfe sich Mitglieder verschiedener gesellschaftlicher Gruppen untereinander verständigen können. Mediale Diskurse sind häufig Interdiskurse, da in ihnen z. B. spezialisierte Wissenschafts-, Rechts- oder Politikdiskurse auftauchen (vgl. Link 2011). Gerade vor dem Hintergrund digitaler zeichenbasierter Datenquellen und der damit verbundenen Multimodalität stellt sich die Frage, wie gängige Verfahren der Diskursanalyse auf die neuen Anforderungen onlinebasierter Kommunikation reagieren (vgl. Meier 2011).

In einer humangeographischen Perspektive ist zudem relevant, wie mit der Kategorie des Raums umgegangen wird. Die Verknüpfung von Diskursen, (Online-)Medien und Räumen wird von immer mehr Studien in den Blick genommen und analytisch hinterfragt (vgl. Bittner und Michel 2013; Bittner et al. 2011; Bauriedl 2007a, 2007b; Gebhardt 2001). Diese greifen wir hier auf: Auf der Grundlage aktueller Debatten zur Besonderheit internetbasierter Daten und Kommunikation gehen wir deren Einfluss auf die Entwicklung der raumbezogenen Mediendiskursanalyse nach und geben einen einführenden Überblick über bisherige Arbeiten in der Humangeographie. Dabei spielen insbesondere auch Überlegungen zu unterschiedlich konzipierten Raumbegriffen eine Rolle, um den "komplexen Zusammenhänge[n] zwischen Materialität, den räumlichen Dimensionen sozialer Praktiken, Sprache und Macht" (Glasze und Matissek 2009, S. 7) gerecht zu werden.

Ergänzt wird dies durch ein empirisches Fallbeispiel: Anhand der vor allem journalistischen Berichterstattung zum jährlich stattfindenden Kunstfestival 48 Stunden Neukölln werden wir eine raumbezogene Kritische Diskursanalyse nach Siegfried und Margarete Jäger (vgl. 1997, 2007) vornehmen. Wir arbeiten die spezifische Bedeutungskonstruktion des Berliner Bezirks Neukölln in 
Interdiskursen heraus und nutzen die klassische Mediendiskursanalyse zumindest erweitert für Überlegungen zur Rolle des Internets z. B. als "Resonanzraum des Diskurses" (Galanova und Sommer 2011, S. 169). Das Festival dient als Blaupause für ein diskursives Ereignis, in dessen Kontext ortsspezifische Bedeutungs- und Wahrheitsproduktionen verhandelt und wirkmächtig werden.

\subsection{Besonderheiten internetbasierter Daten}

Zunächst ist festzuhalten, dass internetbasierte Daten als Kommunikationsdaten bedeutsam für die sozialwissenschaftliche Raumforschung sind. Gleichwohl ist vor allem für qualitative Ansätze eine deutliche Zurückhaltung festzustellen, Online-Medien zu verwenden und zu analysieren (vgl. Schirmer et al. 2015). Während einerseits die Verfügbarkeit von Daten im und aus dem Netz groß ist, existieren andererseits, im Vergleich etwa zu traditionellen Medien, keine institutionalisierten

Archivierungspraktiken; die Publikationspraktiken sind häufig unregelmäßig und insgesamt ist die Kommunikation eher dezentral organisiert: Themen entwickeln sich wenig systematisch und entfalten sich außerdem in Teilöffentlichkeiten. Das heißt, sie werden z. B. „aus traditionellen Massenmedien aufgenommen (...) [und] in Blogs, Wikis oder Social Networks diskursiv weiterverarbeitet" (Galanova und Sommer 2011, S. 169) oder machen spezifische (häufig wenig formalisierte) Selektions- und Ausschlusskriterien wirksam. Für die Forschung mit internetbasierten Daten folgt daraus, dass plausible Kriterien für und gegen die Auswahl der Daten darzulegen bzw. zu entwickeln sind. Gerade die multimediale Vernetztheit der Daten macht ihre Auswahl kritisch und im Forschungsprozess schwierig.

Im Zusammenhang mit dem Internet als Forschungskontext geht es also um die Art und Menge der dort verfügbaren Daten, aber auch um deren Dokumentationsformen, mögliche Erhebungs-, Auswertungs- und Verarbeitungsverfahren. Ganz konkret stellen sich dabei technische oder methodische Fragen: Wie kann bei der Auswahl internetbasierter Daten und Medien vorgegangen werden? Wie lassen sich deren Inhalte analysieren? Wie weit führen gängige Methoden, wo liegen deren Grenzen? Gleichzeitig sind aber auch wissenschafts- bzw. erkenntnistheoretische Fragestellungen relevant. In Bezug auf qualitative Verfahren stellt sich etwa die Frage, wie Daten entstehen und wie dies bei der Rekonstruktion von subjektivem Sinn zu berücksichtigen ist (vgl. Schirmer et al. 2015).

In jüngerer Zeit sind zudem einige Forschungsarbeiten entstanden, die sich unter dem Stichwort "geoweb" mit der medialen Durchdringung des Alltags und damit verbundenen Raumerfahrungen und Praktiken der Raumerschließung auseinandersetzen (vgl. Gryl et al. 2013). Das Internet, besonders das Web2.0, werden hier zu einer "lebensweltlich wirksame[n] und wirkmächtige[n] Größe" (ebd., S. 12) und es setzen sich neue Formen der Geovisualisierung durch, etwa in der Form kollaborativer Kartenerstellungen. Damit verändert sich auch die geographische Wissensproduktion, denn "Modi und Regeln für Aushandlungs- und Entscheidungsprozesse werden hierbei beständig online reorganisiert. Das Monopol der Produktion von Wissen, Wertungen und Deutungen wird auf diese Weise demontiert, nicht nur der Zugang, sondern auch die Kommunikation von Information wird demokratisiert" (ebd., S. 14).

An der Produktion von Diskursen, die sich mit Räumlichkeit beschäftigen, sind nun immer mehr Agierende beteiligt und klare Grenzen zwischen "Produzierenden" und "Konsumierenden" werden aufgelöst. In der Forschung gibt es Positionen, die hierin die erwähnte "Demokratisierung kartographischer Produktion" (Bittner und Michel 2013, S. 111) sehen, während andere die in den "neuen Praktiken und Prozessen ebenso konflikthafte[n] und macht-volle[n] Aushandlungen,

Sichtbarmachungen und Unsichtbarmachungen, Einschlüsse und Ausschlüsse" (ebd.) betonen. Diese verschiedenen Perspektiven machen deutlich, dass sich im Laufe der vergangenen Jahre zwar die technischen Möglichkeiten rasant entwickelt haben, die Grundfragen des Verhältnisses von Diskursen, (Online-)Medien und Räumen, aber ähnlich geblieben sind.

\subsection{Eine humangeographische Perspektive}

Die Diskursanalyse ist in ihren Varianten zwar ein etabliertes methodisches Verfahren in der Geographie, setzt sich aber häufig nicht explizit mit dem Raumverständnis auseinander. Im Gegensatz dazu wird Raum im Kontext des spatial turn als relational, prozesshaft und sozial konstruiert konzipiert 
(vgl. z. B. Miggelbrink 2014; Glasze und Mattissek 2009; Mattissek 2007; Miggelbrink 2002). So ist es notwendig, innerhalb der Diskursanalyse unterschiedliche Raumkonzeptionen ( $u$. a. Belina und Michel 2011, Dünne und Günzel 2006) zu berücksichtigen, um die weitreichenden Wechselwirkungen und Konstitutionen des sozialen Raumes abbilden zu können. Dieser Zusammenhang zwischen Räumlichkeit und Macht ist im Mittelpunkt des Interesses der geographischen Diskursforschung:
"Denn wenn man konzeptionell anerkennt, dass zum einen Räume nicht einfach gegeben sind, sondern immer neu konstituiert werden, und zum anderen die Verfasstheit von Räumen ein wichtiges Element der Herstellung sozialer Wirklichkeit ist, dann ist die Konstitution bestimmter Räume eng verknüpft mit der hegemonialen Durchsetzung bestimmter sozialer Wirklichkeiten" (Glasze und Matissek 2009, S. 12f.).

Hinsichtlich der zugrunde gelegten Diskurskonzepte und des methodischen Vorgehens wird zunehmend differenziert. Humangeographische Studien operationalisieren so grundlegende Anforderungen an ein diskursanalytisches Vorgehen ganz unterschiedlich und behandeln dabei diverse geographische Forschungsgebiete. Für ein Vorgehen, das insbesondere den medialen Diskurs in Blick nehmen will, halten wir dabei die folgenden empirischen Studien für "inspirierend":

\section{Räumliche Anordnungsmuster in stadtentwicklungspolitischen Diskursen}

Sybille Bauriedl (2007a, 2007b) analysiert auf der Grundlage von "klassischen“ Medien - z. B. lokalen Leitdokumenten der Stadtentwicklung, des Parlaments und der Lokalpresse - Nachhaltigkeitsdiskurse in der Hamburger Stadtentwicklung. Die Autorin plädiert für eine stärkere "Raumsensitivität" in der Diskursforschung (Bauriedl 2007b, S. 280 ff.). Diese verstehe Raum entweder als "Sozialgefüge", "konkreten Ort", "Diskurslandschaft" oder "Maßstabsebene” (ebd., S. 280). Bauriedl schlägt einen multimethodischen (und dabei gleichzeitig sehr aufwändigen) Ansatz vor, der die "räumliche Relationalität" (ebd., S. 304) von Diskursen mit in den Blick nimmt. Auf lokaler Ebene lassen sich Diskursfragmente von Lokalitäten z. B. über Kartierungen spezifisch verorten. In dieser Studie werden insbesondere Diskursstruktur und -ordnung, aber auch diskursive Ereignisse und lokaler Diskurskontext über mediale Dokumente auf einer Maßstabsebene empirisch erfassbar - und damit wiederum in Bezug zu räumlichen Konstruktionen und Konstitutionen gesetzt.

\section{Diskursive Verräumlichungen in Printmedien}

Den Zusammenhang von Raum, Wissen und Macht nehmen Paul Reuber und Anke Strüver $(2009,2011)$ als Ausgangspunkt, um die diskursive Verwendung von geopolitischen Leitbildern in deutschen Printmedien (SZ, FAZ, taz, Spiegel) unmittelbar nach dem 11. September mit quantitativen und qualitativen Verfahren zu untersuchen. Sie betonen, wie in den Medien ein "räumlicher Reflex" (Reuber und Strüver 2009, S. 319) nachvollzogen werde: So würden "die Ereignisse mit Hilfe geopolitischer Repräsentationen und Stereotype auch für die Alltagsbetrachtung in Form eines geographisch lokalisierbaren ,Eigenen' und ,Fremden' repräsentiert" (ebd., S. 319). Mit dem Fokus auf die Dekonstruktion von solchen geopolitischen Rhetoriken, Leitbildern und Metaphern, wie die Medien sie nach den Anschlägen des 11. September verbreiteten, können Reuber und Strüver nachzeichnen, welche Rolle territorialisierende Zuschreibungen abhängig vom Verlauf der Auseinandersetzungen bzw. der Krise einnehmen.

\section{Visuell-digitale Repräsentationen globaler Konflikte}

Lisa Parks' Studie (2009) führt besonders deswegen weiter, weil sie nicht nur einen "klassischen" Mediendiskurs in den Blick nimmt, sondern mit der digitalen Plattform Google Earth ein "neueres" Medium. Ausgangspunkt bildet das Projekt Crisis in Darfur (initiiert vom United States Holocaust Memorial Museum, Google Earth; vgl. zum Projekt selbst ebd.). Interessant sind im Verhältnis von Raum, Diskurs und (Online-)Medien nicht nur die "Verräumlichungen" von Informationen und neue Formen der vernetzten Raumrepräsentation. Relevant ist gerade auch das widersprüchliche Verhältnis vom Projekt Crisis in Darfur auf der Plattform Google Earth und dem Presse-Diskurs: Die internationale Presse, so die Autorin, habe positiv auf das Projekt reagiert. Der Konzern Google sei gelobt und ihm humanitäre Absichten zugesprochen worden. Eine genauere Analyse der Datenbank selbst enthüllt allerdings durchaus kritische Aspekte dieser digitalen Konfliktkarten. Während, zugespitzt, die Presse 
Crisis in Darfur als "humanitäres Instrument" (ebd., S. 437) feierte, analysiert Parks die Plattform eher als "Archiv" (ebd., S. 444) des Konflikts. Die kartographische Bereitstellung und Aufbereitung von solchen Krisenereignissen mithilfe neuer Anwendungs- und Verbreitungsmöglichkeiten über das Web2.0 bietet damit nur auf den ersten Blick den Vorteil von Dynamik, Veränderbarkeit - und mithin sogar Partizipation. Auf den zweiten Blick und vor dem Hintergrund der Relationen von Medien, Raum und Diskurs sind auch solche Karten eingebettet in gesellschaftliche Produktions- und Transformationsprozesse, "in denen sich die Beziehungen zwischen Staat, Privatwirtschaft und zivilgesellschaftlichen Akteuren verschieben" (Bittner et al. 2011, S. 64).

Der Zusammenhang von Raum, Medien und Diskursen wird unterschiedlich operationalisiert und forschungspraktisch umgesetzt. Was aber bedeuten diese methodisch-konzeptionellen Überlegungen für die eigene Forschungspraxis? Anhand eines konkreten Beispiels werden wir im Folgenden einige Aspekte aufgreifen, forschungspraktisch erproben und diskutieren. Auch in unserer eigenen Untersuchung beziehen wir uns in erster Linie auf klassische Printmedienformate, die aber auch online verfügbar sind und dort rezipiert werden.

\section{4 .}

\section{Das Fallbeispiel: Berlins Bezirk Neukölln im Spannungsfeld von Medien, Diskurs und Raum}

Das Projekt ist im Zusammenhang mit dem IfL ExperiSpace Das Internet als (neue) Datenquelle für die raumbezogene Forschung (IfL Forschungswerkstatt \#1, Leipzig, Februar 2015) entstanden. Ziel des ExperiSpaces war, aktuelle forschungsbezogene Debatten zum Thema raumbezogene (Online)Medienanalysen in Beziehung zu mediendiskursanalytischen Verfahren zu setzen und diese am Beispiel Berlin Neukölln praxisbezogen zu erproben. Anhand der journalistischen Berichterstattung über das jährlich stattfindende Kunstfestival 48 Stunden Neukölln sollte die spezifische Bedeutungskonstruktion des Berliner Bezirks in einem Interdiskurs herausgearbeitet und in Hinblick auf den vorgestellten Rahmen (Medien - Raum - Diskurs) diskutiert werden. Im Anschluss an die Forschungswerkstatt haben wir - aufgrund der zeitlichen Begrenzung der etwa fünfstündigen Veranstaltung - die ersten

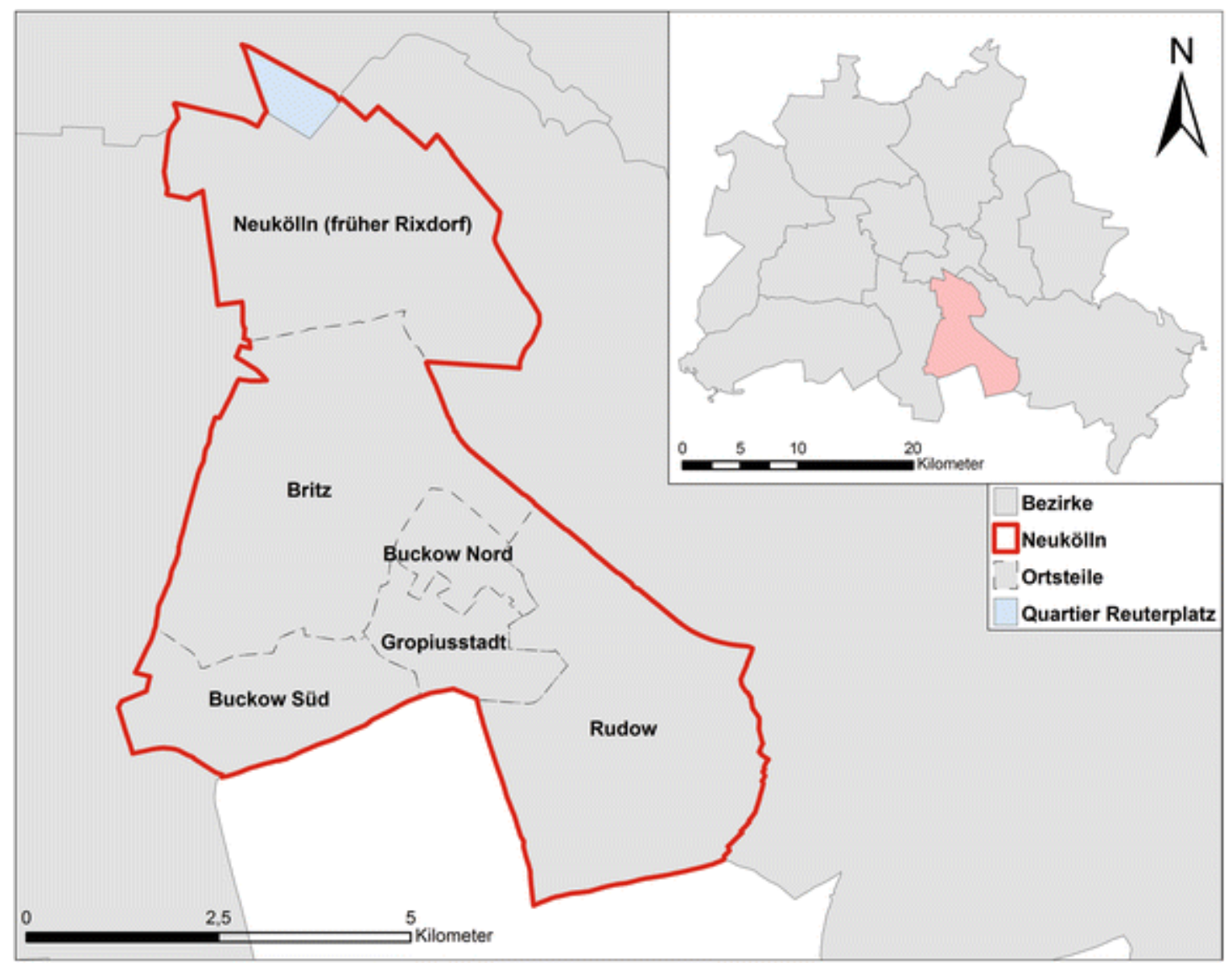

Abb.1 Der Bezirk Neukölln in Berlin und seine Ortsteile 
fragmentarischen Auswertungsansätze weiter ausgearbeitet und für den vorliegenden Aufsatz um zusätzliche Analysen ergänzt.

Zunächst folgt ein Überblick über die jüngeren historischen, für unsere Argumentation konstitutiven Entwicklungen Neuköllns1, bevor erste Ergebnisse der Diskursanalyse vorgestellt werden. Dabei unterliegt auch die historische Betrachtung einer diskursanalytischen Logik bzw. kann keinesfalls als außerhalb des Diskurses - als quasi "natürliche" und "wahre" Geschichte - betrachtet werden. Hier schließen wir uns der Position der Kritischen Diskursanalyse an, die postuliert, dass gesellschaftliche Wirklichkeit zu deuten immer auch heißt, die (historische) Positionalität der jeweils Deutenden in die Analyse mit einzubeziehen (vgl. Jäger und Jäger 2007).

\section{"Endstation Neukölln"?}

Der Berliner Bezirk Neukölln geht auf vier mittelalterliche Dörfer (Rixdorf, Britz, Buckow, Rudow) zurück. Insbesondere Rixdorf entwickelte zunehmend einen gewerbliches Profil und wurde ab Mitte des 19. Jahrhunderts zu einer durch Industrie geprägten Berliner Vorstadt (vgl. Escher 1988). Die Namensänderung in Neukölln 1912 sollte dem Image eines "schmutzigen Arbeiterortes" entgegenwirken - was jedoch scheiterte (vgl. ebd.). Durch die Vereinigung zu Groß-Berlin 1920 wurden Neukölln und die drei angrenzenden Gemeinden in Berlin eingegliedert und bilden seitdem den Bezirk Neukölln (vgl. Bienert und Buchholz 2005).

Auch nach dem Zweiten Weltkrieg blieb Neukölln ein wichtiger Industriestandort innerhalb Berlins. Der Zuzug von sogenannten Gastarbeitenden nach West-Berlin (vor allem aus der Türkei), führte auch in Neukölln zu einem enormen Anstieg ausländischer Bevölkerung (vgl. Kessinger 2012), deren Anteil in der Folge von 12.000 (1972) auf 34.000 Einwohnende (1980) anwuchs. Ab Ende der 1970er-Jahre entstand so eine migrantisch orientierte sozio-kulturelle Infrastruktur. So wurde 1978 das erste türkische Kultur-und Bildungszentrum Berlins gegründet und bis Ende der 1980er-Jahre etablierten alle größeren Migrantinnen- und Migrantengruppen in Neukölln eigene Organisationen (vgl. ebd. S. 143ff.). Die nach der Wiedervereinigung in Berlin einsetzende wirtschaftliche Transformation wirkte sich besonders auf die migrantische Bevölkerung aus, die nach wie vor mehrheitlich in der Industrie tätig war und deren Arbeitslosenquote um etwa 50 \% höher als die der deutschen Bevölkerung lag (vgl. SenStadtUmTec 1995). Der 1997 erschienene SPIEGEL-Artikel "Endstation Neukölln” (vgl. Wensierski 1997), der dem Bezirk den sozialen Niedergang attestierte, kann in diesem Zusammenhang als mediales Schlüsselereignis in der diskursiven Bedeutungszuschreibung des Bezirkes als Problemgebiet gesehen werden (vgl. Gebhardt 2001). Auch aktuell ist der Bezirk durch soziale und städtebauliche Probleme gekennzeichnet. Von den derzeit in Berlin existierenden 37 Quartieren, die durch das

Städtebauförderprogramm Die Soziale Stadt: Stadtteile mit besonderem Entwicklungsbedarf unterstützt werden, liegen allein elf in Neukölln - die meisten, betrachtet man alle zwölf Berliner Bezirke (vgl. SenStadtUm 2015).

Die mediale Debatte um Neukölln als „Problembezirk” erreichte 2006 ihren Höhepunkt, als im Reuterkiez Schulkinder Lehrpersonal tätlich angriffen (vgl. von Randow 2006). Neukölln galt nun als Paradebeispiel für eine als gescheitert angesehene Integrationspolitik in Deutschland (vgl. Sutterlüty 2006). Die noch häufig unsanierte Bausubstanz und ein massiver Gewerbeleerstand im Gebiet um Nord-Neukölln unterstützten die Genese des negativen Images und wirkten damit letztlich stigmatisierend auf den gesamten Bezirk (vgl. Holm 2011). Im Rahmen eines vom Quartiersmanagement Reuterkiez beauftragten Projektes entwickelten sich insbesondere Unternehmen der Kreativwirtschaft als wichtige Neumieter und schufen $66 \%$ der 200 neu entstehenden Arbeitsplätze (vgl. Brammer 2008). Darüber hinaus zogen vermehrt Studierende zu und es siedelten sich mehr Bars und Kneipen an. Folglich bewerteten die Medien (Nord-)Neukölln zunehmend als Szene-Bezirk. Das Berliner Magazin ZITTY titelte in diesem Zusammenhang bereits 2008: "Neukölln rockt. Mit zitty unterwegs in Berlins derzeit spannendstem Bezirk" (vgl. Zitty 2008).

1 Wie auf Abb. 1 zu sehen, ist Neukölln sowohl Berliner Bezirk als auch Ortsteil des gleichnamigen Bezirkes. Um Verwechslungen zu vermeiden, wird im Folgenden „Neukölln“ für die Bezeichnung des Bezirkes und „Nord-Neukölln“ in Anlehnung an Gößwald und Schmiedeknecht (2009) sowie Holm (2011) für den Ortsteil genutzt 
Zeitgleich entwickelte sich Nord-Neukölln zwischen 2007 und 2013 als der Berliner Stadtteil, in dem die durchschnittlichen Mieten am meisten stiegen (10,5 \%) (vgl. ImmobilienScout24 2014). Der Aufwertungsprozess in Neukölln ist jedoch im Vergleich mit anderen Hauptstadtbezirken, in denen durch Sanierungsprogramme physische Aufwertungen am Gebäudebestand erfolgten, durch eine symbolische Gentrifikation gekennzeichnet, bei dem das Image des Szene-Bezirks der eigentlichen Aufwertung vorausgeht (vgl. Holm 2011). Das 2010 vom Reiseportal der New York Times gezeichnete Bild des „hippen“ Neukölln (vgl. McGrane 2010) ist darüber hinaus auch Ausdruck der zunehmenden Internationalisierung von Bewohnerschaft und Laufpublikum in Neukölln.

Diese Konstruktion Neuköllns zwischen den beiden Polen Problemviertel und Szene-Bezirk ist dabei im besonderen Maße im jährlich stattfindenden Kunstfestival 48 Stunden Neukölln eingeschrieben. So wurde das Festival 1999 als Reaktion auf eine anhaltende negative Berichterstattung über den Bezirk ins Leben gerufen, um kulturell und künstlerisch einen Gegenpol zur diskursiven Abwertung zu bilden (vgl. 48 Stunden Neukölln 2015). Das Festival findet seitdem jedes Jahr im Sommer statt, ist stetig gewachsen und mittlerweile an mehr als 300 Spielorten vornehmlich in Nord-Neukölln vertreten. Die folgenden Ausführungen beleuchten, welches Bild von Berlin-Neukölln im Rahmen der journalistischen Berichterstattung und ihrer Online-Rezeption zu 48 Stunden Neukölln zwischen 2006 und 2014 generiert und diskutiert wird. Diese Analyse wird ergänzt um eine Betrachtung der Veranstalterbeiträge des Festivals auf der Mikrobloggingplattform Twitter.

\section{Stunden Neukölln im medialen Diskurs - Fallbeispiel und Methodik}

Um das Meinungsbild einer möglichst breiten Bevölkerungsschicht einzufangen und die Diskursanalyse auf der Ebene der alltäglichen Wissensproduktion anzusetzen, wurden für das Fallbeispiel medial vermittelte Interdiskurse in den Blick genommen (vgl. Gebhardt 2001). Dabei wurde die Zeitspanne von 2006 - aufgrund der Vorkommnisse an der Rütli-Schule eine der Hochphasen der landesweit negativen Berichterstattung über Berlin-Neukölln - bis Ende 2014 gewählt, um den Wandel in der medialen Zuschreibung Neuköllns vom "Problembezirk" hin zum "Szene-Bezirk" beleuchten und nachvollziehen zu können.

Dem Internet als Datenquelle kommt hierbei für die mediale Berichterstattung eine zweifache Bedeutung zu: Einerseits fungiert es für Zeitungsartikel, die auch in Printmedien veröffentlicht wurden, als Speichermedium und Distributionsplattform. Andererseits ist es im Rahmen von Lesendenkommentaren und Text- oder Bildnachrichten (z.B. über Twitter, Facebook, YouTube, etc.) Produktionsort diskursiver Aussagen. Die nun folgende Mediendiskursanalyse berücksichtigt daher beide Quellenarten. Forschungspragmatisch ist hier zu erwähnen, dass sich online verfügbare Zeitungsberichte im Vergleich zu gedruckten Beiträgen recht zeiteffizient erheben, selektieren, speichern und auswerten lassen.

Die Online-Archive von Berliner Tageszeitungen, Wochenzeitungen, Boulevardzeitungen und Stadtmagazine wurden zunächst nach den Stichwörtern "48 Stunden Neukölln" innerhalb des genannten Zeitraumes durchsucht. Bei den meisten (Wochen)Zeitungen oder Magazinen reichten die Treffer für eine sinnvolle Analyse über einen Zeitraum von acht Jahren nicht. Zugleich ist die mediale Berichterstattung zu 48 Stunden Neukölln ist sehr lokal geprägt und findet darüber hinaus fast nicht statt. Die Beiträge mussten folgenden drei Charakteristika genügen, um als relevant eingestuft und einer weiteren Analyse unterzogen zu werden:

1. Die Berichterstattung muss sich zentral mit dem Ereignis 48 Stunden Neukölln beschäftigen, eine bloße Nennung von Veranstaltungen im Rahmen des Kunstfestivals reicht nicht aus.

2. Der Ort Neukölln muss qualitativ mit Bedeutungszuweisungen konstruiert werden.

3. Dabei ist irrelevant, ob diese Bedeutungszuweisungen direkt von der Autorin oder dem Autor kommen oder beispielsweise im Rahmen eines Interviews oder Zitates gewählt wurden.

Lediglich bei den drei größten Berliner Tageszeitungen gab es eine ausreichend hohe Zahl an relevanten Artikeln: Berliner Zeitung, Der Tagesspiegel und Berliner Morgenpost. Dort fanden sich insgesamt 29 Artikel und zwei Videos, die dann mit den dazugehörigen Lesendenkommentaren einer 
Diskursanalyse unterzogen wurden.

Diese wurde nach Jäger und Jäger (vgl. 2007, 1997) durchgeführt, welche die Verfassende als methodisches Verfahren gut ausgearbeitet und Schritt für Schritt beschrieben hat. Das Verfahren ist eng an die Diskurstheorie Michel Foucaults angelehnt und wurde entwickelt, um "diskursive Sagbarkeitsfelder darzustellen, diese zu interpretieren und einer Kritik zu unterziehen" (ebd. S. 15). Dabei werden ein Diskursstrang oder mehrere miteinander verschränkte Diskursstränge sowohl in ihrer historischen Entwicklung als auch gegenwartsbezogen untersucht - durchaus mit dem Ziel, Aussagen über die mögliche zukünftige Entwicklung von Diskursen zu generieren (vgl. Jäger und Jäger 1997). Ein Diskursstrang bezeichnet thematisch einheitliche Diskursverläufe, die aus mehreren Aussagen - den sogenannten Diskursfragmenten - bestehen. Diese Fragmente beinhalten die für die Diskursstränge typischen Themen (vgl. ebd.).

Nach Auswahl der Beiträge wurde das Material mittels einer Strukturanalyse aufbereitet. Diese verortet die Artikel in den jeweiligen Diskurssträngen, erfasst angesprochene und fehlende Themen und versucht Einblick in die Diskurspositionen sowie Standpunkte der jeweiligen Zeitungen und Autorinnen und Autoren zu geben. In der dann folgenden Feinanalyse wurden die Form und Struktur von Diskurssträngen, ihre Kontextbezüge, sprachlich-rhetorischen Merkmale sowie inhaltlich-ideologischen Aussagen anhand ausgewählter, besonders dichter und stellvertretend stehender Beiträge (bzw. Diskursfragmente) analysiert und dechiffriert (vgl. Jäger und Jäger 2007). Da der Schwerpunkt dieses Aufsatzes auf der Diskussion methodischer und analytischer Implikationen im Zusammenhang mit Medien, Diskurs und Raum liegt, werden nachfolgend die Ergebnisse der Analyse nur kurz zusammengefasst.

\section{Der Online-Printmediendiskurs: Kunst und Kultur werten den "Problembezirk" auf}

Die ausgewählten Beiträge zu 48 Stunden Neukölln zeichnen ein Bild des Bezirks, das von der Fülle seiner Zuschreibungen her ambivalent erscheint und sich insbesondere an folgenden vier miteinander verwobenen Diskurssträngen orientiert: Multikulturalität, Kreative und Kunstschaffende, Problembezirk und Szene-Bezirk. In frühen Berichten (etwa 2006 bis 2010) orientieren sich die Beiträge an den Bezugspunkten Multikulturalität und verschiedenen Lebensstilen. Sie verweisen darauf, dass Kultur in Neukölln für Außenstehende etwas Ungewöhnliches sei, dabei aber untrennbar mit der Multikulturalität sowie den Problemen im Bezirk zusammenhinge. Es wird der Schluss gezogen, dass die heterogene Mischung des Bezirks gleichzeitig der Nährboden für kreativen Freiraum darstelle. Dabei wird die Funktion, die Kreative und Kunstschaffende in der möglichen Aufwertung spielen, herausgehoben und Neukölln im weiteren Verlauf der Berichterstattung zum Szene-Bezirk und Kreativquartier der Stadt erklärt. Soziale Probleme des Bezirks rücken mehr und mehr in den Hintergrund. In den Medienbeiträgen ab etwa 2010 treten dann verstärkt ökonomische Argumentationsmuster auf: Neukölln wird als attraktiver Standort für kulturelle und kreativwirtschaftliche Aktivitäten beschrieben, begünstigt durch immaterielle (Atmosphäre, Netzwerke) und materielle (günstige Mieten, Freiräume) Aspekte. Auf diese Weise wird gleichzeitig ein Verstetigungs- und Gentrifizierungsprozess beschrieben, der unweigerlich in einem Strukturwandel enden werde. Neukölln als "Problembezirk" wird im Rahmen dieser Zuschreibungen eher als Blaupause eines schon überwundenen Zustands diskutiert. Nur stellenweise wird eingeräumt, dass es immer noch soziale Probleme gibt. Das vergangene Neukölln wird als "verrucht" oder "sozial verarmt, kriminell und heruntergekommen" beschrieben (Deckwerth 2011) - und dient damit als Antonym zum heutigen Neukölln, in dem es eine große, lebendige und internationale Szene von Kreativen und Kunstschaffenden gebe, die den ehemaligen "Problembezirk" "salonfähig" (ebd.) gemacht habe.

\section{Der Rezeptionsdiskurs: Von "elitären" Gruppen und dem "Schmuddelkiez"}

Die Auswertung von Lesendenkommentaren zu den Artikeln über 48 Stunden Neukölln zeigt, dass es kaum Reaktionen auf diese Beiträge gibt. Die geringe Zahl von Kommentaren ist allerdings vergleichbar mit denen zu anderen Berichten in den drei ausgewählten Zeitungen, die sich ebenfalls lokalpolitischen Themen widmen. Das enthüllt eventuell ein generelles methodisches Problem, das den Zugang zu lokalen Rezeptionsdiskursen kennzeichnet: Von den insgesamt 29 Artikeln bekamen nur zwei Kommentare und zwar jeweils sechs. Deren Analyse kann nur erste vorsichtige Hinweise geben, 
inwiefern sich Rezeptionsdiskurse auf die Diskursstruktur der medialen Darstellung beziehen: Einerseits hinterfragen bzw. negieren die Kommentierenden die Rolle von Kunstschaffenden und Kreativen im Zusammenhang mit einer positiven Stadtteilentwicklung. Vielmehr thematisieren sie eine mögliche Gefahr, die von einer solchen Aufwertung ausgehen könnte und negative Folgen für die Sozialstruktur Neuköllns hätte. Auf der anderen Seite greifen die Kommentare das Thema Neukölln als "Problembezirk" auf, das - anders als in der neueren medialen Berichterstattung postuliert lebensweltlich durchaus weiterhin von sozialen und materiellen Problemen dominiert werde, etwa was Vermüllung oder Verwahrlosung betrifft. Hier wird die soziale Aufwertung und Gentrifizierung Neuköllns durch zugezogene Kunstschaffende und Intellektuelle sozialkritisch in den Blick genommen und Neukölln parallel dazu als verwahrloster und heruntergekommener Kiez beschrieben.

\section{Der Twitter-Diskurs: „Kunst rettet Welt”}

In der erweiterten Datenerhebung zur Online-Rezeption von 48 Stunden Neukölln wurden neben den Medienberichten und den Online-Kommentaren auch Quellen aus sozialen Medien berücksichtigt. Konkret haben wir die Auseinandersetzung mit Neukölln auf Twitter durch die Veranstaltenden des Kunstfestivals ausgewertet. Auf deren offizieller Twitter-Seite (@48hNk) waren zum Auswertungszeitpunkt (bis November 2015) 923 Tweets abrufbar. Bei deren Analyse zeigte sich, dass sich die Mehrzahl davon nicht konkret auf Neukölln bezieht. Vielmehr widmen sich die Tweeds einer großen Bandbreite an Themen: Neben gesellschaftspolitischen Statements finden sich auch konkrete Veranstaltungshinweise zu Kunst und Kultur, Retweets anderer Beiträge oder Interaktionsaufrufe.

Im deutlichen Gegensatz zur Berichterstattung der Print- bzw. Massenmedien, welche die Kunst- und Kreativszene Neuköllns - hier in Gestalt des Festivals - sinnbildlich für Aufwertungsprozesse behandeln und mit Globalisierung und Ökonomisierung in Zusammenhang bringen, folgen die analysierten Tweets aus der Innensicht der Agierenden einer gänzlich anderen Logik: Sie verstehen das Festival, aber auch Kunst im Allgemeinen Is eine mögliche Form des sozialpolitischen Engagements. Dies äußert sich auch deutlich im Leitthema des Festivals von 2015: "S.O.S. - Kunst rettet Welt". Auch Kunstschaffenden wird eine andere Rolle zugeschrieben: Statt sie als Treibende einer lokalen städtischen Aufwertung zu betrachten, sehen die Veranstaltenden des Festivals sie eher als mögliche Verlierende von Aufwertungsprozessen ("Gentrifizierung betrifft auch Künstler_innen berlinweit", 5. September 2015). Die Rolle von Kunst und Kunstschaffenden in Interaktion mit dem Ort, an dem sie stattfindet, wird dabei als kollektiver Aushandlungsprozess begriffen. Dies zeigt sich auch in den rhetorischen Mitteln: per direkter Anrede ("Wenn ihr wissen wollt, ..." "Wollt ihr von Neukölln ...?" , "... hat eine Bitte an euch!") wird die Leserschaft der Nachrichten aktiv in die Kommunikation eingebunden - und damit auch in die gemeinsame Gestaltung des Bezirks.

Insgesamt wird 48 Stunden Neukölln auf Twitter als kritisch-intellektuell eingebunden in eine größere Kunstszene präsentiert. Dabei setzt sich das Festival mit lokalen Problemen auseinander und reagiert vor allem in Form konkreter Veranstaltungen, bezieht sich aber auch auf übergeordnete gesellschaftspolitische Themen.

\subsection{Analyse von raumbezogenen Online-Diskursen: Potenziale und Herausforderungen}

Das Internet kann im Zusammenhang mit diskursanalytischen Verfahren allgemein als "Resonanzraum eines Diskurses" (Galanova und Sommer 2011, S. 169) bezeichnet werden. Einerseits werden Diskurse in diesem Raum gespeichert und verbreitet - z. B. als online verfügbare Beiträge aus klassischen Printmedien. Andererseits werden solche Diskurse aber auch diskursiv rezipiert, aufbereitet und weiterverbreitet. Damit gehen Spezifika für die raumbezogene Forschung einher: Wenn Bauriedl (2007b) in ihrer Studie zu Hamburger Nachhaltigkeitsdiskursen nach dem Verhältnis von physischem und sozialem Raum fragt, muss diese Frage im Zusammenhang mit Online-Daten noch um den digitalen Raum ergänzt werden. „Das Lokale entsteht [...] im Wechselspiel mit globalen Prozessen und Narrativen" (ebd., Abs. 27) - diese Aussage, mit der Bauriedl auf die Notwendigkeit einer multimethodischen, diskursanalytischen Mehrebenenperspektive verweist, wird im Zusammenhang mit im Internet produzierten, modifizierten, gespeicherten und rezipierten Zeichen(-Systemen) nochmals erweitert (vgl. ebd., Abs. 36). 
Internetbasierte Kommunikation ist notwendigerweise in gesamtgesellschaftliche Diskurse eingebunden, gleichwohl sind die Inhaltsproduktion und -rezeption im Netz durch eine komplexe, netzartige Verweisstruktur gekennzeichnet. In vielen Fällen - der oben analysierte Twitter-Diskurs ist aufgrund seiner relativ eindeutigen Autorenschaft als Ausnahme anzusehen - sind nicht nur die Rollen, die unterschiedliche Agierende wie die Online-Portale der Printzeitungen im Kommunikationsprozess einnehmen, kaum eindeutig zu beschreiben. Auch die Fallauswahl ist methodisch nur mit einigem Aufwand zu bewerkstelligen. In unserem Fallbeispiel ist es z. B. grundsätzlich nicht ausgeschlossen, dass bestimmte, uns aufgrund mangelnden Wissens oder fehlender Involviertheit verschlossene Teilöffentlichkeiten andere wirkmächtige Diskurse führen - Foren etwa, Blogs oder Social-MediaGruppen,

Vorteilhaft für die raumsensible Diskursforschung in der Arbeit mit digitalen Daten ist hingegen, dass sie vergleichsweise einfach zugänglich sind. So lassen sich vor allem Diskursverläufe, die sich über lange Zeit erstrecken, anhand von z. B. Schlüsselbegriffen zielstrebig und vergleichsweise mühelos identifizieren. Gleichzeitig liefert der Fokus auf die Multimodalität der Daten im Netz bzw. insbesondere die Kombination von Bild- und Textzeichen Hinweise auf die Konstituierung von Raum. Bauriedl (vgl. 2007a) spricht hier am Beispiel der Hamburger Hafen-City davon, wie symbolische Konstruktionen und Inszenierungen auf materielle Strukturen konkreter Orte einwirken - sowie umgekehrt. Unser Fallbeispiel legt ebenfalls eine (Re-)Präsentation des Materiellen im Diskursiven nahe wie auch eine (Re)Produktion in umgedrehter Richtung.

\subsection{Ergänzung diskursanalytischer Verfahren um multimethodische Ansätze}

Die Verknüpfung solcher symbolisch-kommunikativen mit materiellen Strukturen ist im Rahmen einer Diskursanalyse nicht einfach zu leisten. Wenn in den vorangegangen Ausführungen vor allem der Diskurs über Neukölln innerhalb der medialen Berichterstattung in den Blick genommen wurde, konnte damit gezeigt werden, inwiefern der Bezirk hier (historisch) "gedacht" bzw. konstruiert wird.

Der Rezeptionsdiskurs, also jener Diskurs, der zeigt, wie Individuen - und damit auch mögliche Bewohnende - Neukölln denken und konstruieren, hat auf der Ebene der Online-Rezeption von Printartikeln bisher nur begrenzt Ergebnisse vorgehalten. Daneben hat die Auswertung der TwitterNachrichten wiederum eine sich deutlich vom Printmediendiskurs unterscheidende Darstellung offengelegt. Um soziale Räume einer umfassenden Analyse zu unterziehen, „also möglichst das gesamte Wissen, das diese Räume hervorgebracht hat und erhält, zu elizitieren" (Jäger und Jäger 2007, S. 275), könnten insbesondere dispositivanalytische Vorgehen umfassendere Einblicke gewähren, die neben Diskursen auch Praktiken und Handlungsweisen der Bewohnenden Neuköllns bzw. der Agierenden im Bezirk sowie "Institutionen und Sichtbarkeiten einbeziehen, die für die Strukturen im Stadtteil wesentlich sind" (ebd., S. 284).

Neben den hier diskutierten klassischen text- und bildbasierten Kommunikationsdaten im und aus dem Web sind im Rahmen raumbezogener Online-Analysen aber auch die im Zusammenhang mit der Studie von Parks (vgl. 2009) genannten digitalen Karten zu betrachten. Besonders durch die Entwicklungen des Web2.0 verändern sich Autorenschaften, Produktions- und Verbreitungsdynamiken, Quantität, Qualität und Validität der georeferenzierten Daten, aber auch die gesellschaftspolitischen Aushandlungsprozesse, die in den Karten eingeschrieben sind. Damit wird also eine kritische Analyse von Web2.0-Karten vor neue methodische Anforderungen gestellt (vgl. Bittner und Michel 2013, Bittner et al. 2011).

Hier im Aufsatz wurde auf den Zusammenhang von raumbezogener Forschung, diskursanalytischen Methoden und online-basierten Daten(-quellen) eingegangen. Während sich wissenschaftliche Debatten immer ausführlicher um die hier behandelten Aspekte drehen, steht die Beantwortung weiterer Fragen noch aus - etwa Fragen, die sich in Verbindung mit der Analyse riesiger, hochauflösender, in Echtzeit generierter Datensets stellen (Stichwort Big Data). Diese werden aber mit großer Wahrscheinlichkeit auch für die raumbezogene Forschung in Zukunft an Relevanz gewinnen. 


\subsection{Literatur}

48 Stunden Neukölln (Hrsg) (2015) Unser Leitbild. http://www.48-stunden-neukoelln.de/de/page/leitbild Zugegriffen: 10. September 2017

Bauriedl S (2007a) Spielräume nachhaltiger Entwicklung. Die Macht stadtentwicklungspolitischer Diskurse. oekom, München

Bauriedl S (2007b) Räume lesen lernen: Methoden zur Raumanalyse in der Diskursforschung. In: FQS 8, H. 2, Art. 13

Belina B, Michel B (2011) Raumproduktionen. Beiträge der Radical Geography. Eine Zwischenbilanz. Westfälisches Dampfboot, Münster

Bienert M, Buchholz E (2005) Die zwanziger Jahre in Berlin: Ein Wegweiser durch die Stadt. Berlin Story, Berlin

Bittner C, Glasze G, Michel B, Turk C (2011) Krisen- und Konfliktkarten im Web 2.0. Geographische Rundschau 11:60-65

Bittner C, Michel B (2013) Das Dekonstruieren der web2.0 Karte. Vorschläge zur Analyse dynamischer und interaktiver Karten multipler und diffuser Autorenschaften. In: Gryl I, Nehrdich T, Vogler R. (Hrsg) geo@web. Medium, Räumlichkeit und geographische Bildung. Springer VS, Wiesbaden, S 111-126

Brammer M (2008) Zwischennutzung in Berlin Neukölln. Kreativwirtschaft als Motor in einem sozial benachteiligten Binnenquartier. In: Standort - Zeitschrift für Angewandte Geographie 32:71-77

Deckwerth S (2011) Luxus im Knast. In Berliner Zeitung, 16.06.2011. http://www.berlinerzeitung.de/newsticker/luxus-im--knast,10917074,10923382.html Zugegriffen: 10. September 2017

Dreesen P, Kumię ga Ł, Spieß C (2012) Mediendiskursanalyse. Diskurse - Dispositive - Medien - Macht. Springer VS, Wiesbaden

Dünne, J, Günzel S (2006) Raumtheorie. Grundlagentexte aus Philosophie und Kulturwissenschaften. Suhrkamp, Frankfurt a. M.

Escher, F (1988) Neukölln. In: Geschichte der Berliner Verwaltungsbezirke. Band 3. Colloquium, Berlin

Fairclough N (1995) Critical Discourse Analysis. Addison Wesley, Boston

Fairclough N (2000) Discourse and social change. Polity Press, Cambridge

Foucault M (1981) Archäologie des Wissens. Suhrkamp, Frankfurt a. M.

Galanova O, Sommer V (2011) Neue Forschungsfelder im Netz. Erhebung, Archivierung und Analyse von Online-Diskursen als digitale Daten. In: Schomburg S, Leggewie C, Lobin H, Puschmann C (Hrsg) Digitale Wissenschaft. Stand und Entwicklung digital vernetzter Forschung in Deutschland. Köln, S 169178

Gebhardt D (2001) "Gefährliche fremde Orte" - Ghetto-Diskurse in Berlin und Marseille. In: Heller W, Asche $\mathrm{H}$, Bürkner H-J (Hrsg) Praxis Kultur- und Sozialgeographie 24. Potsdam

Glasze G, Mattissek A (2009) Handbuch Diskurs und Raum: Theorien und Methoden für die Humangeographie sowie die sozial- und kulturwissenschaftliche Raumforschung. Transcript, Bielefeld

Gößwald U, Schmiedeknecht K (Hrsg) (2009) Wie zusammen leben - Perspektiven aus Nord-Neukölln: ein Kooperationsprojekt der Quartiermanagements Schillerpromenade, Rollbergsiedlung, Körnerpark und Flughafenstraße mit dem Kulturamt Neukölln und dem Museum Neukölln. Dokumentation der Ausstellung vom 22. Juni 2008 bis 28. Juni 2009 im Museum Neukölln. Berlin

Gryl I, Nehrdich T, Vogler R (2013): geo@web. Zur Entfaltung und Anverwandlung eines neuen Forschungsfeldes In: Gryl I, Nehrdich T, Vogler R. (Hrsg) geo@web. Medium, Räumlichkeit und geographische Bildung. Springer VS, Wiesbaden, S 9-31

Holm A (2011) Gentrification in Berlin: Neue Investitionsstrategien und lokale Konflikte. In Herrmann H, Keller C, Neef R, Ruhne R (Hrsg) Die Besonderheit des Städtischen. Entwicklungslinien der 
Stadt(soziologie). Springer VS, Wiesbaden, S 213-232

ImmobilienScout24 (Hrsg) (2014) Kaltmieten_Berlin_BGID. Datensatz zur Entwicklung der Angebotsmieten in Berliner Ortsteilen von 2007-2013. Berlin

Jäger S (2009) Kritische Diskursanalyse. Eine Einführung. Unrast, Münster

Jäger S, Jäger M (1997) Bemerkungen zur Durchführung von Diskursanalysen. Duisburger Institut für Sprach- und Sozialforschung. http://www.diss-

duisburg.de/Internetbibliothek/Artikel/Durchfuehrung_Diskursanalyse.htm Zugegriffen: 10. September 2017

Jäger S, Jäger M (2007) Deutungskämpfe - Theorie und Praxis Kritischer Diskursanalyse. Springer VS, Wiesbaden

Kessinger B (2012) Neukölln. Die Geschichte eines Berliner Stadtbezirks. Vergangenheitsverlag, Berlin Läpple D (1991) Essay über den Raum. Für ein gesellschaftswissenschaftliches Raumkonzept. In: Häußermann $\mathrm{H}$ et al. (Hrsg) Stadt und Raum. Soziologische Analysen. Centaurus, Pfaffenweiler, S 157207

Link J (2011) Diskursanalyse unter besonderer Berücksichtigung von Interdiskurs und Kollektivsymbolik. In: Keller R, Hirseland A, Schneider W, Viehöver W: Handbuch Sozialwissenschaftliche Diskursanalyse. Band 1: Theorien und Methoden. Springer VS, Wiesbaden, S 108-130

Lossau J, Lippuner R (2004) Geographie und Spatial Turn. In: Erdkunde 58:201-211

Mattissek A (2007) Diskursanalyse in der Humangeographie - "State of the Art". In Geographische Zeitschrift 95:37-55

McGrane S (2010) Berlin, A Creative Wave. In: New York Times Travel.

http://www.nytimes.com/slideshow/2010/10/24/travel/20101024-surfacing-berlin.html?_r=0 Z Zugegriffen: 10. September 2017

Meier S (2011) Multimodalität im Diskurs. Konzept und Methode einer multimodalen Diskursanalyse In: Keller R, Hirseland A, Schneider W, Viehöver W: Handbuch Sozialwissenschaftliche Diskursanalyse. Bd 1: Theorien und Methoden. Springer VS, Wiesbaden, S 499-532

Miggelbrink J (2002) Der gezähmte Blick. Zum Wandel des Diskurses über "Raum" und „Region” in humangeographischen Forschungsansätzen des ausgehenden 20. Jahrhunderts. Institut für Länderkunde, Leipzig

Miggelbrink J (2014) Diskurs, Machttechnik, Assemblage. Neue Impulse für eine regionalgeographische Forschung. In Geographische Zeitschrift 102:25-40

Parks L (2009) Ausgrabungen in Google Earth. Eine Analyse der ,Darfur-Krise'. In: Döring J, Thielmann T (Hrsg) Mediengeographie. Theorie - Analyse - Diskussion. Transcript, Bielefeld, S 431-454

Randow von G (2006) Unter Polizeischutz. Ein Einwanderungsland ist entsetzt wegen seiner Probleme: Berlin-Neukölln ist kein Einzelfall. Eine Nachrichtenanalyse. In: Zeit-Online, 05.04.2006. http://www.zeit.de/online/2006/14/ruetlischule Zugegriffen: 10. September 2017

Reuber P, Strüver A (2009) Diskursive Verräumlichungen in deutschen Printmedien. Das Beispiel Geopolitik nach 9/11. In: Döring J, Thielmann T (Hrsg) Mediengeographie. Theorie - Analyse Diskussion. Transcript, Bielefeld, S 31-332

Reuber P, Strüver A (2011) Der Anschlag von New York und der Krieg gegen Afghanistan in den Medien. Eine Analyse der geopolitischen Diskurse. In: Dzudzek I, Reuber P, Strüver A (Hrsg) Die Politik räumlicher Repräsentation. Beispiele aus der empirischen Forschung. LIT, Berlin, S 197-216

Rothfuß E, Dörfler T (2013) Prolog - Raumbezogene Qualitative Sozialforschung. Konzeptionelle Überlegungen zwischen Geographie und Soziologie. In: Dies. (Hrsg) Raumbezogene Qualitative Sozialforschung. Springer VS, Wiesbaden

Schirmer D, Sander N, Wenninger A (2015) Die qualitative Analyse internetbasierter Daten. Methodische Herausforderungen und Potenziale von Online-Medien. Springer VS, Wiesbaden 
SenStadtUm (Senatsverwaltung für Stadtentwicklung und Umwelt Berlin) (Hrsg) (2015) Soziale Stadt, Quartiersmanagement - Karte der Gebiete.

http://www.stadtentwicklung.berlin.de/wohnen/quartiersmanagement/de/karte.shtml Zugegriffen: 10.

September 2017

SenStadtUmTec (Senatsverwaltung für Stadtentwicklung, Umweltschutz und Technologie Berlin) (Hrsg) (1995) Migration. Berlin: Zuwanderung, gesellschaftliche Probleme, politische Ansätze. BerlinSutterlüty F (2006) Dynamik der Gewalt. Wie Ohnmachtsgefühle sich in einen Machtrausch verkehren können. Der Fall der Neuköllner Rütli-Schule. In Zeit-Online, 06.04.2006. http://www.zeit.de/2006/15/Gewalt Zugegriffen: 10. September 2017

Wensierski P (1997) Endstation Neukölln. In Der Spiegel 43. http://www.spiegel.de/spiegel/print/d8805068.html Zugegriffen: 10. September 2017

Zitty (Hrsg) (2008) Neukölln rockt. Mit zitty unterwegs in Berlins derzeit spannendstem Bezirk. Zitty, Das Hauptstadtmagazin, H. 6. http://reuterkiez.net/2008/03/11/zitty-neukoelln-rockt/ Zugegriffen: 10. September 2017

\section{Analysierte Mediendokumente}

48 Stunden Neukölln (Hrsg) (2015) Twitter @48hnk, https://twitter.com/48hnk Zugegriffen: 31. Oktober 2016

Bartels G (2007) „48 Stunden Neukölln“ Kunst mit Seeblick. Der Tagesspiegel, 21. Juni 2007

Bartels G (2008) Kulturfestival. Harmonien im Hausflur. Der Tagesspiegel, 20. Juni 2008

Bartels G (2009) Wochenende. Kunst- und Kulturfestival in Neukölln. Der Tagesspiegel, 26. Juni 2009

Bartels G (2010) Klingeln, bis die Kunst kommt. 48 Stunden Neukölln feiert Rixdorf. Der Tagesspiegel, 24. Juni 2010

Bartels G (2011) Berlin. Für Joop ist Neukölln wie einst New York. Designer unterstützt Festival "48 Stunden". Der Tagesspiegel, 9. Juni 2011.

Berliner Morgenpost (Hrsg) (2007) 48 Stunden Kunst in Neukölln. Berliner Morgenpost, 19. Juni 2007

Berliner Morgenpost (Hrsg) (2010) Kunst: 48 Stunden Neukölln erleben. Berliner Morgenpost, 25. Juni 2010

Berliner Morgenpost (Hrsg) (2011) Das Kunst- und Kulturfestival "48 Stunden Neukölln“. Berliner Morgenpost, 16. Juni 2011

Berliner Morgenpost (Hrsg) (2014) 48 Stunden Neukölln. Berliner Morgenpost, 26. Juni 2014

Bernau N (2008) Erfolg der Notwendigkeit. Berliner Zeitung, 12. November 2008

Deckwerth S (2011) Luxus im Knast. Berliner Zeitung, 16. Juni 2011

Demnitz J (2013) Kunstfestival in Neukölln. Der Tagesspiegel, Video

http://www.tagesspiegel.de/berlin/video-zum-kunstfestival-in-berlin-neukoelln-48-stunden-kunst/8354288.html 14. Juni 2013. Zugegriffen: 31. Oktober 2016

Der Tagesspiegel (Hrsg) (2010) 48 Stunden Neukölln. Der Tagesspiegel, Video

http://www.tagesspiegel.de/suchergebnis/videos/?sw=48+Stunden+Neuk\%C3\%B6IIn\&search fromday $=1 \&$ search - frommonth $=1 \&$ search - fromyear $=2006$ \&search - today $=31$ \&search tomonth=12\&search-toyear=2014, 3. Februar 2010. Zugegriffen: 31. Oktober 2016

Garrelts N (2011) Stark im Schwarm. Der Tagesspiegel, 20. Juni 2011

Janovsky S (2008) Das Kunstfestival 48 Stunden Neukölln zeigt den Bezirk als neue Heimat der Avantgarde. Aber das hat seine Grenzen. Hirsch und Heimat. Berliner Zeitung, 23. Juni 2008

Kiesmann M (2010) Zum Kulturspektakel "48 Stunden Neukölln“ kamen 70000 Leute - Sie schauten sich Höfe an und hörten fremde Geschichten. Selbstgedichtetes für Trinker. Berliner Zeitung, 28. Juni 2010 
Langer L (2013) Kulturfestival "48 Stunden Neukölln". Ein ganzes Wochenende Kunst in Neukölln. Der Tagesspiegel, 13. Juni 2013

Leber S (2006) Berlin knallt hart. Der Tagesspiegel, 23. Juni 2006

Schmiemann B (2010) Neukölln feiert seine Geschichte. Berliner Morgenpost, 27. Juni 2010

Schmidl K (2014) Die Lage hat sich dramatisch verändert. Berliner Zeitung, 26. Juni 2014

Stache R (2008) Utopische Gegenwelten. Berliner Morgenpost, 27. Februar 2008

Strauss S (2007) Wo Kunst ist, kann kein Getto sein. Berliner Zeitung, 25. Juni 2007

Strauss S (2008a) Festival am Wochenende mit Aktionen an 171 Orten. Für 48 Stunden wird Neukölln Künstlerbezirk. Berliner Zeitung, 17. Juni 2008

Strauss S (2008b) 48 Stunden Neukölln. Kunst im Kiez. Berliner Zeitung, 21. Juni 2008

Strauss S (2009) Das Festival "48 Stunden Neukölln" ist an seine Grenzen gestoßen. Alle machen Kunst. Berliner Zeitung, 29. Juni 2009

Strauss S (2010) Das Festival 48 Stunden Neukölln stößt an seine Grenzen. Deshalb gelten jetzt strenge Regeln. Alle wollen Künstler sein. Berliner Zeitung, 17. April 2010

Strauss S (2012a) Kunstfestival 48 Stunden Neukölln. Endstation Paradies in Neukölln. Berliner Zeitung, 11. Juni 2012

Strauss S (2012b) Kunstfestival 48 Stunden Neukölln. Performance im Knast. Berliner Zeitung, 14. Junin 2012

Strauss S (2012c) Kunstfestival "48 Stunden Neukölln”. Kunst auch im Süden Neuköllns. Berliner Zeitung, 20. Juni 2012

Suljkanovic D (2014) Nur die Galerien fehlen noch. Berliner Morgenpost, 28. Juni 2014

Zylla G (2007) Neukölln wird heute zur Kulturmeile. Berliner Morgenpost, 23. Juni 2007 